\section{Ehab M. Kamel}

Elena Rizzo

Michel A. Duchosal

Rafael Duran

Vasco Goncalves-Matoso

Pierre Schnyder

Salah D. Qanadli

\title{
Radiological profile of anemia on unenhanced MDCT of the thorax
}

\begin{abstract}
Our aim was to investigate the diagnostic value of unenhanced MDCT in anemic patients. Fifty consecutive patients with proven anemia and 50 nonanemic matched group for age, sex and body mass index were evaluated. In either group, hemoglobin levels were assessed no more than $24 \mathrm{~h}$ from an unenhanced CT of the thorax. For each patient, the presence of a hyperattenuating aortic wall (aortic ring sign) and/or dense interventricular septum (subjective parameters) were identified by two radiologists who were blinded to the laboratory findings. Furthermore, the aortic $\mathrm{CT}$ attenuation values (objective parameter) were also obtained and correlated with the hemoglobin levels. The sensitivity and specificity in detecting anemia were calculated for each variable, and ROC analysis was generated for subjective and objective parameters. Subjective image analysis revealed that the aortic ring sign was more sensitive than the interventricular septum sign for anemia detection
\end{abstract}

( $84 \%$ vs. $72 \%)$, whereas this latter sign was more specific $(100 \%$ vs. $92 \%)$. A good correlation $(r=0.60)$ was observed between the aortic CT attenuation values and the hemoglobin levels in the whole study population. Using a threshold of $\leq 35 \mathrm{HU}$ for anemia diagnosis, the sensitivity and specificity of aortic CT attenuation value were $84 \%$ and $94 \%$, respectively, with the largest area under the curve (0.89) among all diagnostic criteria. However, the best trade-off between sensitivity $(80 \%)$ and specificity $(100 \%)$ was obtained from combining both subjective and objective analysis. Interpreting anemia upon unnenhanced MDCT of the thorax is quite feasible. A diagnostic approach that considers both subjective and objective analysis offers the best trade-off between sensitivity and specificity.

Keywords Anemia - Unenhanced CT $\cdot$ Thorax

\section{Introduction}

Incidental findings on thoracic CT are common [1]. Despite their wide range of clinical relevance, identifying such incidental findings may impose considerable impact on patient management and outcome $[2,3]$. Some investigators have previously reported the incidental detection of severe anemia on unenhanced CT of the thorax [4-8]. Distinguishing the myocardium or interventricular septum as two relatively dense structures from a hypodense left ventricular cavity has been claimed to be a reliable sign for the diagnosis of anemia [4-8]. This approach, however, has been recently shown to be an operator-dependant process that may be subject to significant inter-observer variability [9]. Furthermore, in some diseases such as secondary hemochromatosis and glycogen storage disease, excess cardiac iron or glycogen may result in a relative increase in the interventricular density despite normal hemoglobin levels $[4,8]$. Similarly, the identification of a hyperattenuating aortic wall has been shown to indicate an underlying 
anemia $[4,5]$. This sign, however, can be easily mimicked by the existence of calcified atherosclerotic plaques. Objective analysis, in contrast, is a more accurate process that can differentiate anemic from nonanemic subjects [9]. This can be established by analyzing the blood pool density of great vessels or cardiac chambers upon unenhanced CT.

Motivated by this background, we decided to investigate the diagnostic potential of subjective and objective analysis of anemia, both separately and combined, on unenhanced $\mathrm{CT}$ of the thorax.

\section{Materials and methods}

\section{Patients}

In our monocentric study, 50 consecutive patients with laboratory-proven anemia who also had unenhanced CT examinations of the thorax for various indications were enrolled. During the same period, which extended from March 2006 to August 2007, another group of 50 nonanemic patients matched for age, sex, and body mass index was also recorded. Thus, the study population consisted of 100 patients, 67 men and 33 women with a mean age of 56 years (age range, 19 to 94 years). The unenhanced CT examinations were performed under the following conditions: research of infectious or inflammatory lung disease in patients with chronic renal insufficiency $(n=20)$, lung biopsy $(n=16)$, aortic CT angiography $(n=15)$, cardiac CT $(n=14)$, follow-up of indeterminate pulmonary nodules $(n=13)$, research of lung metastasis in patients with renal insufficiency $(n=7)$, research of hemorrhagic source $(n=5)$, follow-up of lung fibrosis $(n=5)$, and CT guided drainage of pleural effusion $(n=5)$. Hemoglobin levels were measured by photometry using a Sysmex XE2100 analyzer (Sysmex Digitana AG, Horgen, Switzerland) on samples collected within $24 \mathrm{~h}$ from respective CT scans. Anemia was defined as a hemoglobin level below $12 \mathrm{~g} / \mathrm{dl}$ for women and below $14 \mathrm{~g} / \mathrm{dl}$ for men [10]. Mild anemia was reported when the hemoglobin level showed value below the normal level for either sex down to $9.5 \mathrm{~g} / \mathrm{dL}$, whereas moderate and severe anemia were reported when the hemoglobin level was $\leq 9.4 \mathrm{~g} / \mathrm{dl}$, but $\geq 8.0 \mathrm{~g} / \mathrm{dl}$ and $\leq 7.9 \mathrm{~g} / \mathrm{dl}$, respectively $[10,11]$. Patients with active bleeding or blood transfusion reported between the determination of the hemoglobin levels and the CT were not included in our analysis. Institutional review board approval was obtained for the present analysis.

\section{CT scan analysis}

The whole study cohort underwent unenhanced CT on a 64-detector CT system (VCT, GEMS, Milwaukee, WI) using $1.25-5 \mathrm{~mm}$ thickness, 1.375 pitch, $180-400 \mathrm{~mA}$, and $120 \mathrm{kVp}$. All images were reconstructed using a standard kernel filter as specified by the manufacturer (STND filter, GEMS). No oral contrast medium was given for any patient either before or during the CT examination. For each individual patient, two radiologists (a general radiologist and a cardiovascular radiologist) with 4 and 15 years of experience identified the presence or absence of (1) hyperattenuating aortic wall "aortic ring sign" against relatively hypodense aortic blood pool and (2) dense myocardium or interventricular septum "interventricular septum sign" against hypodense left ventricular cavity (Fig. 1). All CT examinations were interpreted in consensus using soft tissue window (window width, $200 \mathrm{HU}$; window level, $75 \mathrm{HU}$ ). Although a typical mediastinal window would necessitate $400-\mathrm{HU}$ window width and $0-40-\mathrm{HU}$ window level, we have purposely chosen modified values to maximize the contrast gradient between the blood pool and both the aortic wall and the interventricular septum as previously described [7]. To avoid any potential interobserver conflicts, patients who displayed equivocal findings were interpreted as nonanemic. Both radiologists were unaware of the laboratory findings. Additionally, blood density was quantified through measuring the $\mathrm{CT}$ attenuation value (HU) by means of regions of interest (ROIs) drawn over the ascending aorta at the level of bifurcation of the main pulmonary artery. Regions of beam-hardening, streak, and motion artifacts were avoided owing to their known detrimental effect on the accuracy of quantitative analysis. No ROIs have been assigned to the left ventricular cavity since its diagnostic yield has been previously shown to be uncertain, especially in nonanemic patients [12]. All ROI measurements were performed by an independent observer blinded to both clinical and laboratory data using a dedicated workstation (Advantage Window 4.2; GEMS). A standard circular ROI measuring $2 \mathrm{~cm}^{2}$ was applied for the whole study cohort.

\section{Statistical analysis}

The sensitivity, specificity, and accuracy of both subjective and objective analysis were calculated both separately and combined. For combined subjective analysis, anemia was reported when both the aortic ring sign and the interventricular septum sign were identified. For combined subjective and objective analysis, anemia was reported when at least $2 / 3$ positive diagnostic criteria were evidenced. The values derived from aortic ROIs of both patient groups are given as mean $\pm \mathrm{SD}$. Variables were compared using the paired t-test; a $P$-value of less than 0.05 was considered significant. Using linear regression analysis, correlation between hemoglobin level and the aortic CT attenuation values was evaluated. Receiver-operating characteristic (ROC) curves were generated for each diagnostic criterion as well as for both subjective and objective analysis 
Fig. 1 Unenhanced CT at the level of main pulmonary artery (a) and the ventricular level (b) in a woman with severe anemia (hemoglobin, $8.4 \mathrm{~g} / \mathrm{dl}$ ) shows both the aortic ring sign (arrows) and the interventricular septum sign (arrow heads). Corresponding sections in nonanemic male patient (hemoglobin, $16 \mathrm{~g} / \mathrm{dl}$ ) display normal appearance of the aortic wall (c) as well as of the myocardium (d)
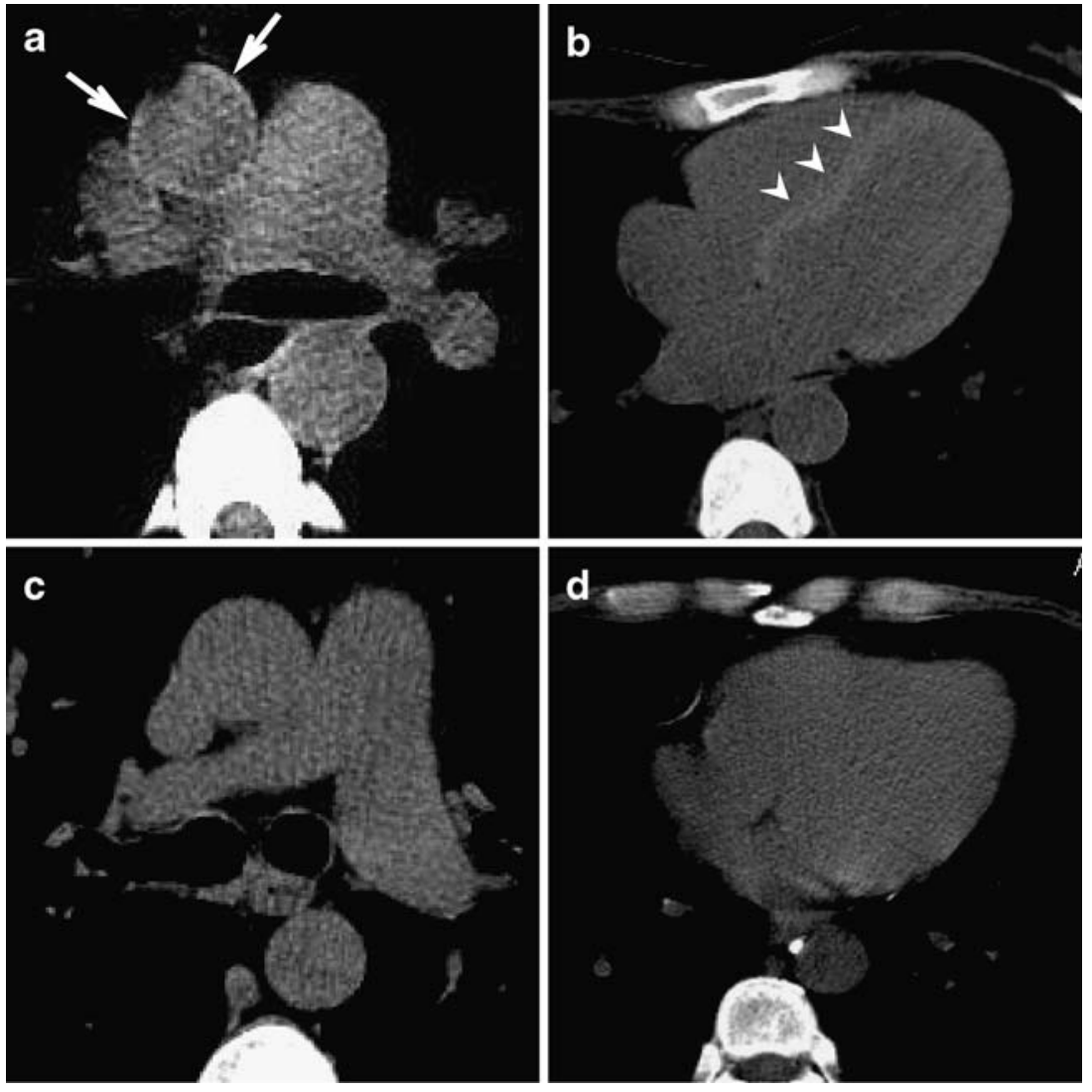

combined to establish the best diagnostic parameter(s) for anemia on unenhanced CT of the thorax.

\section{Results}

Laboratory assessment of hemoglobin values for the anemic group $(\mathrm{n}=50)$ ranged from 6 to $12.9 \mathrm{~g} / \mathrm{dl}$ (mean, $10 \pm 1.5 \mathrm{~g} / \mathrm{dl}$ ). Corresponding values for the nonanemic group $(\mathrm{n}=50)$ ranged from 12 to $16.5 \mathrm{~g} / \mathrm{dl}$ (mean, $14 \pm$ $0.9 \mathrm{~g} / \mathrm{dl}$ ). Four patients had severe anemia (mean, $6.6 \pm$ $0.6 \mathrm{~g} / \mathrm{dl}$ ), whereas moderate and mild anemia were reported in 11 (mean, $8.8 \pm 0.3 \mathrm{~g} / \mathrm{dl}$ ) and 35 (mean, $10.8 \pm 0.9 \mathrm{~g} / \mathrm{dl}$ ) patients, respectively. Subjective image analysis revealed that the aortic ring sign was more sensitive than the interventricular septum sign in anemia detection, whereas this latter sign was more specific (Table 1). The dense interventricular septum sign was negative in 14 of the 35 (40\%) patients who presented with mild anemia (mean hemoglobin, $11.4 \pm 0.9 \mathrm{~g} / \mathrm{dl})$. No patient with moderate $(\mathrm{n}=$ 11) or severe $(n=4)$ anemia has been missed. Of these 14 patients, $6(43 \%)$ displayed positive aortic ring sign (mean hemoglobin, $11 \pm 0.7 \mathrm{~g} / \mathrm{dl}$ ) (Fig. 2). In the remaining 8 of 14 $(57 \%)$ patients, anemia (mean hemoglobin, $11.9 \pm 0.9 \mathrm{~g} / \mathrm{dl}$ ) was missed since none of the aforementioned radiological signs had been perceived.

The existence of minute calcification in atherosclerotic mural plaques of the aorta was responsible for falsepositive diagnosis of anemia in 4/50 (8\%) nonanemic patients (mean age, $62 \pm 4$ years) who displayed factitious aortic ring sign (Fig. 3). These four patients, who did not present with symptoms or signs suggestive of inflammatory aortic wall disease or had been identified with aortic mural thrombus upon unenhanced CT examination revealed at least one macroscopic mural calcification (Fig. 3).
Table 1 Performance of the three diagnostic parameters (aortic CT attenuation value, aortic ring sign, and interventricular septum sign) in detecting anemia, both separately and combined

\begin{tabular}{llll}
\hline Diagnostic parameter(s) & Sensitivity & Specificity & Accuracy \\
\hline Aortic ring sign & $84 \%$ & $92 \%$ & $88 \%$ \\
Interventricular septum sign & $72 \%$ & $100 \%$ & $86 \%$ \\
Aortic CT attenuation value ( $\leq 35 \mathrm{HU})$ & $84 \%$ & $94 \%$ & $89 \%$ \\
Combined subjective analysis & $72 \%$ & $100 \%$ & $86 \%$ \\
Combined subjective and objective analysis & $80 \%$ & $100 \%$ & $90 \%$ \\
\hline
\end{tabular}


Fig. 2 Unenhanced $\mathrm{CT}$ at the level of main pulmonary artery (a) and the ventricular level (b) in a man with mild anemia (hemoglobin, $12 \mathrm{~g} / \mathrm{dl}$ ) shows true-positive aortic ring sign (arrows) against false-negative interventricular septum sign
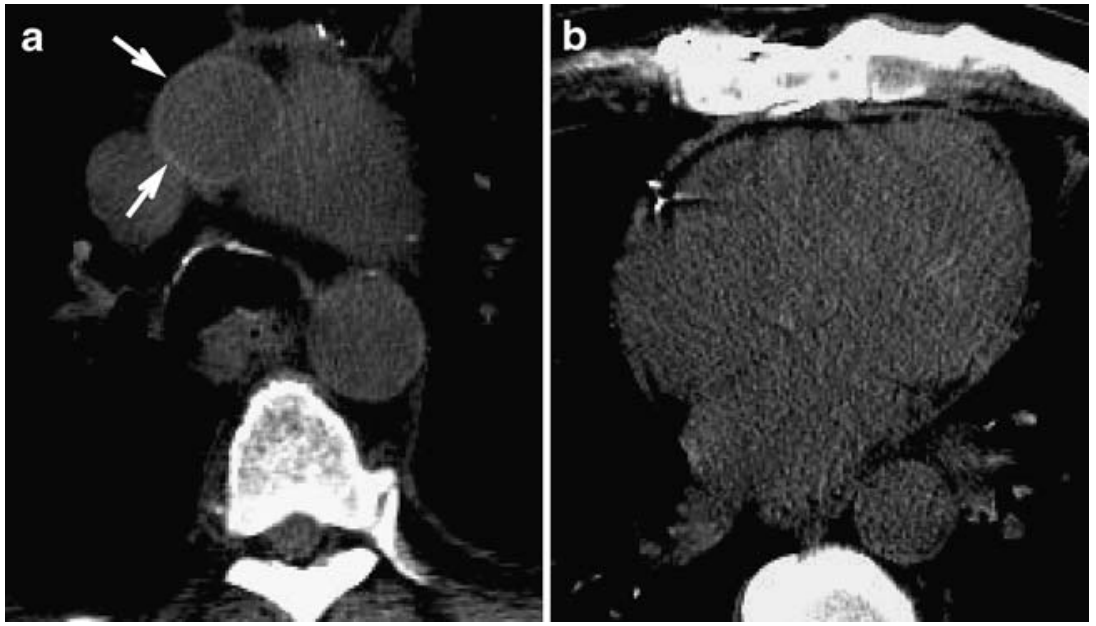

On the other hand, no false-positive results were associated with the application of the interventricular septum sign since we did not encounter any patient with either iron or glycogen storage disease in our series.

The data derived from ROIs analysis revealed a significant difference between the aortic CT attenuation values in anemic and nonanemic groups (range, 14 to $42 \mathrm{HU}$, mean, $28 \pm 7 \mathrm{HU}$ vs. 30 to $56 \mathrm{HU}$, mean, $41 \pm$ $5.5 \mathrm{HU}, P<0.0001)$. A significant positive correlation $(r=$ 0.60 ) was found between the aortic $\mathrm{CT}$ attenuation values and hemoglobin levels in both patient groups (Fig. 4). ROC curve analysis revealed that the best threshold for anemia diagnosis using the aortic $\mathrm{CT}$ attenuation values was $\leq 35 \mathrm{HU}\left(\mathrm{A}_{\mathrm{Z}}=0.89\right)$. This threshold successfully identified $42 / 50$ anemic patients and ruled out anemia in $47 / 50$ nonanemic patients. Corresponding values for the aortic ring sign, the dense interventricular septum sign, and combined analysis (subjective and objective) were $\mathrm{A}_{\mathrm{Z}}=$ $0.88, \mathrm{~A}_{\mathrm{Z}}=0.86$, and $\mathrm{A}_{\mathrm{Z}}=0.90$, respectively (Fig. 5). Worth noting is that we observed a non-specific zone of transition ( $\geq 31 \mathrm{HU}$ to $\leq 42 \mathrm{HU}$ ) between mild anemia and normal hemoglobin levels in $11 / 100(11 \%)$ patients with $(n=8$, mean hemoglobin $11 \pm 1.5 \mathrm{~g} / \mathrm{dl})$ and without $(\mathrm{n}=3$, mean hemoglobin $13.5 \pm 0.5 \mathrm{~g} / \mathrm{dl}$ ) anemia. Descriptive statistics revealed that the best trade-off among sensitivity, specificity, and accuracy were obtained from analyzing both subjective and objective data combined (Table 1).

\section{Discussion}

The prevalence of anemia has been recently shown to be more common than is generally considered [11]. Besides its well-known prevalence among oncology and critical care patients, the incidence of anemia among aged people is four to six fold greater than generally suspected [11, 13]. Recognition and treatment of anemia are important since untreated anemia carries increased risks of morbidity, mortality, and long hospital stay. These can indeed augment the cost of health-care delivery [11]. However, despite these facts, anemia remains a neglected and undertreated diagnosis [11]. Consequently, every effort must be done to identify and treat anemia as early as possible to avoid many unnecessary comorbidities.
Fig. 3 Unenhanced CT at the level of main pulmonary artery (a) in a nonanemic man (hemoglobin, $15 \mathrm{~g} / \mathrm{dl}$ ) shows falsepositive aortic ring sign (arrows) presumably induced by faint calcified atherosclerotic mural plaques. The non-visualization of the interventricular septum sign (b) is a true-negative finding

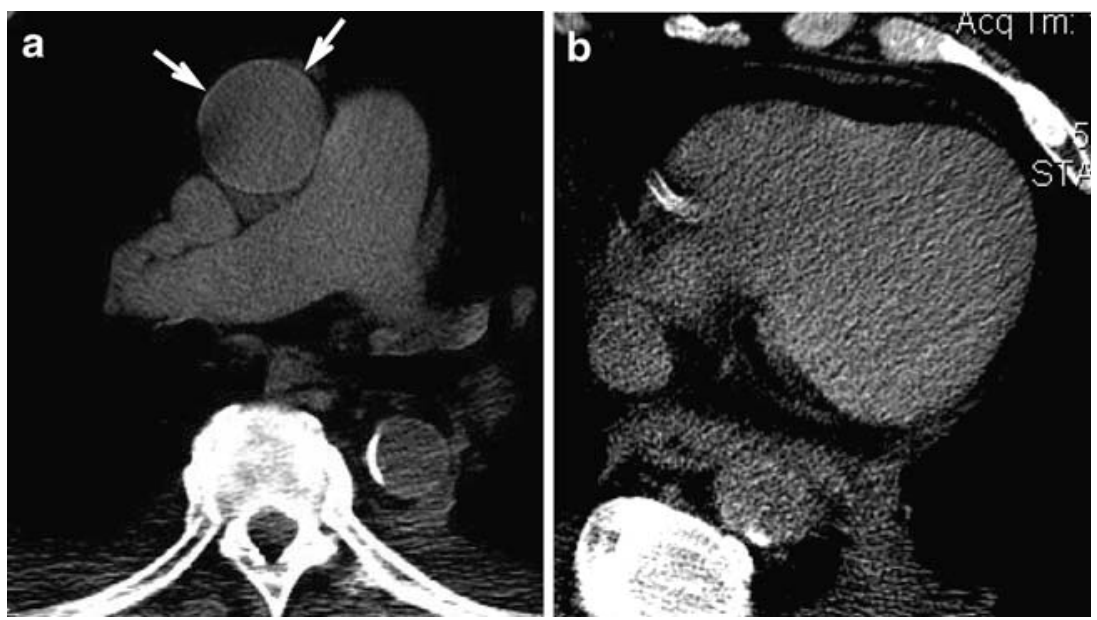




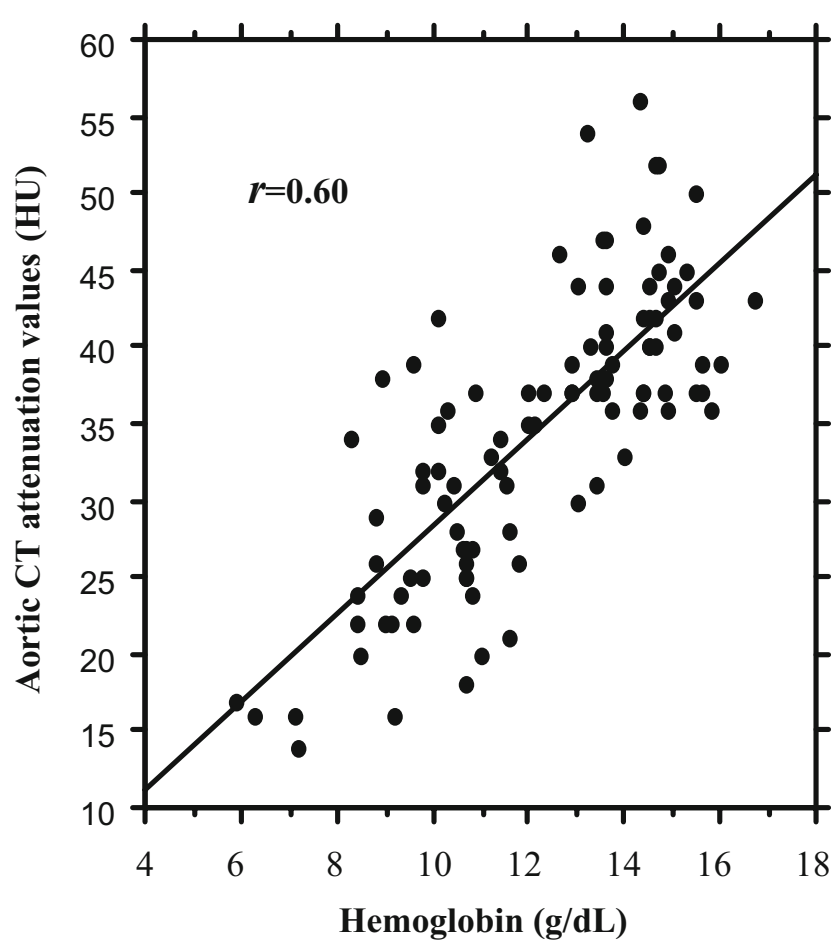

Fig. 4 Graph shows moderate and significant correlation between the aortic CT attenuation values and hemoglobin levels of the whole study population $(\mathrm{n}=100)$

Despite the fact that the majority of modern day CT acquisition protocols apply IV injection of contrast medium; however, there are often a few images obtained before the triggering of the injection that are not routinely interrogated for the potential existence of anemia or other incidental $\mathrm{CT}$ abnormalities. In the present report, we highlight the integral roles of both subjective and objective radiological analysis for detecting anemia on unenhanced CT of the thorax. Despite a specificity of $100 \%$ for the dense interventricular septum, this radiological sign revealed a modest sensitivity of $72 \%$ since it showed false-negative results in $14 / 35(40 \%)$ patients who presented with mild anemia (Fig. 2). This modest sensitivity is not surprising since our strict diagnostic approach denied the diagnosis of anemia in all patients who displayed equivocal findings, a common source of inaccuracy and poor interobserver agreement [9]. In contrast, the aortic ring sign revealed far better sensitivity (84\%) against suboptimal (92\%) specificity. The higher sensitivity figure of the aortic ring sign can be reasoned by better contrast between the aortic wall, which is composed mainly of collagen and elastic fibers, and the aortic blood pool. The myocardium, by comparison, is primarily built of less attenuating muscle fibers (Fig. 2). On the other hand, the lower specificity of the aortic ring sign can be explained by the existence of faint calcification within pre-existing atherosclerotic mural plaques, which are known by their dense appearance upon unenhanced CT (Fig. 3). Other less frequent aortic abnormalities such as Takayasu's arteritis and intramural aortic hematoma may induce similar effects [14]. Identifying any calcified atherosclerotic mural plaques can thus invalidate the diagnostic significance of the aortic ring sign, whereas the smooth, uniform, and homogeneous appearance, and the absence of periaortic fat infiltration may be used as reliable exclusion criteria for any potential inflammatory or other disorder of the aortic wall [15-17].

Quantitative analysis of the aortic CT attenuation values revealed a significant difference between the $\mathrm{HU}$ values of aortic blood pool in anemic and nonanemic patients (mean, $28 \pm 7 \mathrm{HU}$ vs. $41 \pm 5.5 \mathrm{HU}, P<0.0001$ ). Furthermore, there was a significant positive correlation between the HU values of the ascending aorta and the hemoglobin levels in both anemic and nonanemic patients (Fig. 4). Using a cutoff value of $\leq 35 \mathrm{HU}$ for aortic blood pool attenuation, $42 / 50$ of the studied patients were correctly classified as being anemic, whereas anemia exclusion was successfully achieved in $47 / 50$ patients yielding a sensitivity and specificity of $84 \%$ and $94 \%$, respectively. Our results concur with those derived from previous studies [6-9]; however, it has to be recognized that quantitative analysis revealed a non-specific zone of transition ( $\geq 31 \mathrm{HU}$ to $\leq 42 \mathrm{HU}$ ) between mild anemia and normal hemoglobin levels in $11 \%$ of the studied patient population. Despite this limitation, analyzing the HU value of the aortic blood pool can be considered as a useful adjunct that could resolve a

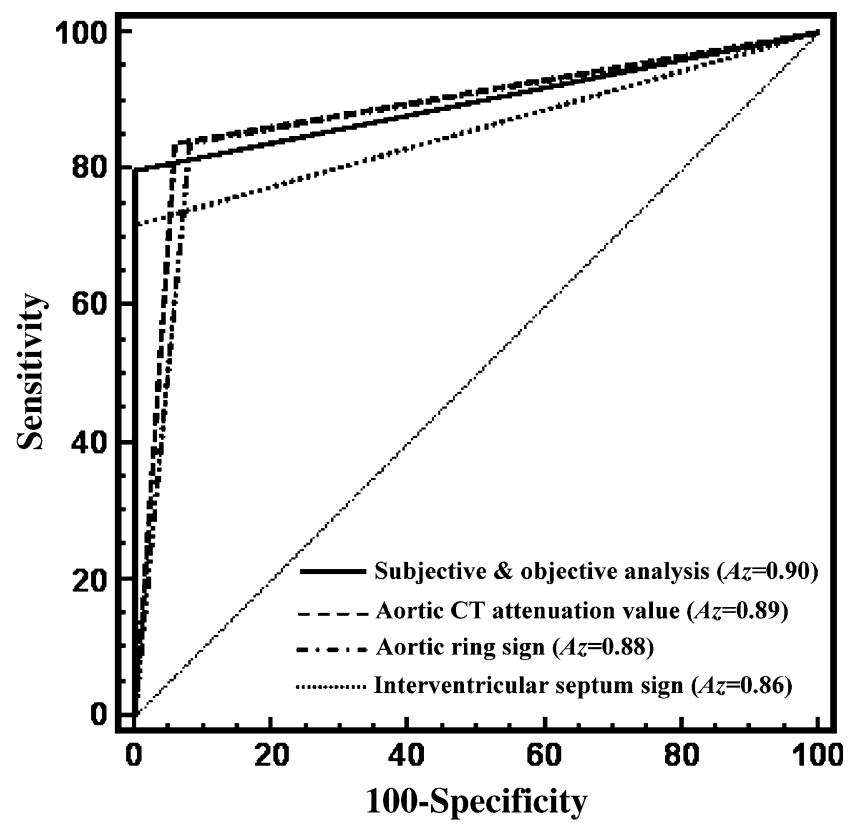

Fig. 5 ROC curves for the three diagnostic parameters (aortic CT attenuation value, aortic ring sign, and interventricular septum sign) along with that for combined subjective and objective analysis and their corresponding area under the curve $\left(A_{z}\right)$ 
wide range of diagnostic uncertainties. This can be strengthened by the results obtained from the ROC curves of the studied diagnostic parameters (Fig. 5). Descriptive statistics revealed that combining both qualitative and quantitative diagnostic parameters offers the best trade-off between sensitivity, specificity and accuracy (Table 1). This implies that a rapid inspection for the aortic ring sign and the dense interventricular septum sign along with measuring the aortic $\mathrm{CT}$ attenuation values may represent the best diagnostic approach to identify anemia upon unenhanced CT of the thorax.

A limitation of the present study may be related to the wide range of X-ray tube currents used for $C T$ in our patient population who had diverse body mass indices. However, applying a weight-adapted scan protocol, as in our series, has been previously shown to yield a high and constant image quality without significant noise contribution $[18$, 19]. Secondly, we could not extend our ROI analysis to include the left ventricular cavity owing to the known absence of contrast gradient between ventricular lumen and interventricular septum in nonanemic patients.

\section{Conclusion}

Interpreting anemia upon unenhanced MDCT of the thorax is quite feasible. A diagnostic approach that considers both subjective and objective analysis offers the best trade-off among sensitivity, specificity and accuracy. Ruling out anemia, in contrast, is currently an uncertain task since the aforementioned diagnostic parameters are not sensitive enough for the minute reductions in hemoglobin levels.

\section{References}

1. van de Wiel JC, Wang Y, Xu DM et al (2007) Neglectable benefit of searching for incidental findings in the DutchBelgian lung cancer screening trial (NELSON) using low-dose multidetector CT. Eur Radiol 17:1474-1482

2. Stillman AE, Oudkerk M, Ackerman M et al (2007) Use of multidetector computed tomography for the assessment of acute chest pain: a consensus statement of the North American Society of Cardiac Imaging and the European Society of Cardiac Radiology. Eur Radiol 17:2196-2207

3. Goetze S, Pannu HK, Wahl RL (2006) Clinically significant abnormal findings on the "nondiagnostic" CT portion of low-amperage-CT attenuation-corrected myocardial perfusion SPECT/CT studies. J Nucl Med 47:1312-1318

4. Doppman JL, Rienmuller R, Lissner J (1981) The visualized interventricular septum on cardiac computed tomography: a clue to the presence of severe anemia. J Comput Assist Tomogr 5:157-160

5. Wojtowicz J, Rzymski K, Czarnecki R (1983) Severe anaemia: its CT findings in the cardiovascular system. Eur $\mathbf{J}$ Radiol 3:108-111
6. Corcoran HL, Cook DE, Proto AV (1988) Diagnosis of anemia on computed tomography scans of the thorax. J Comput Tomogr 12:116-121

7. Collins AJ, Gillespie S, Kelly BE (2001) Can computed tomography identify patients with anaemia? Ulster Med J 70:116-118

8. Foster M, Nolan RL, Lam M (2003) Prediction of anemia on unenhanced computed tomography of the thorax. Can Assoc Radiol J 54:26-30

9. Title RS, Harper K, Nelson E, Evans T, Tello R (2005) Observer performance in assessing anemia on thoracic CT. AJR Am J Roentgenol 185:1240-1244

10. Groopman JE, Itri LM (1999) Chemotherapy-induced anemia in adults: incidence and treatment. J Natl Cancer Inst 91:1616-1634

11. Spence RK (2007) Medical and economic impact of anemia in hospitalized patients. Am J Health Syst Pharm 64 (16 Suppl 11):S3-S10

12. Powell WJ, Wittenberg J, Dinsmore RE, Miller SW, Maturi RA (1977) Definition of cardiac structures using computerized tomography in isolated arrested and beating canine hearts. Am J Cardiol 39:690-696

13. Ania BJ, Suman VJ, Fairbanks VF, Rademacher DM, Melton LJ 3rd (1997) Incidence of anemia in older people: an epidemiologic study in a well defined population. J Am Geriatr Soc 45:825-831
14. Shiotani S, Kohno M, Ohashi N et al (2002) Hyperattenuating aortic wall on postmortem computed tomography (PMCT). Radiat Med 20:201-206

15. Sharma S, Sharma S, Taneja K, Gupta AK, Rajani M (1996) Morphologic mural changes in the aorta revealed by $\mathrm{CT}$ in patients with nonspecific aortoarteritis (Takayasu's arteritis). AJR Am J Roentgenol 167:1321-1325

16. Chung JW, Park JH, Im JG, Chung MJ, Han MC, Ahn H (1996) Spiral CT angiography of the thoracic aorta. Radiographics 16:811-824

17. Castaner E, Andreu M, Gallardo X, Mata JM, Cabezuelo MA, Pallardo Y (2003) CT in nontraumatic acute thoracic aortic disease: typical and atypical features and complications. Radiographics 23:S93-S110

18. Mulkens TH, Bellinck P, Baeyaert M et al (2005) Use of an automatic exposure control mechanism for dose optimization in multi-detector row CT examinations: clinical evaluation. Radiology 237:213-223

19. Kemper J, Regier M, Bansmann PM et al (2007) Multidetector CT urography: experimental analysis of radiation dose reduction in an animal model. Eur Radiol 17:2318-2324 